\title{
European Green Deal und deutsche Energiewende zusammen denken!
}

Der European Green Deal ist in seiner Bedeutung für die deutsche Energiewende nicht zu unterschätzen. Nachdem sich die Staats- und Regierungschefs der EU im Dezember 2019 für eine klimaneutrale EU bis 2050 ausgesprochen haben, soll der European Green Deal eine umfassende Wachstumsstrategie unter Einbindung aller Sektoren - Energiewirtschaft, Gebäude, Industrie, Verkehr und Landwirtschaft - entwickeln und Maßnahmenpakete zur Ressourcenschonung und Emissionsminderung implementieren. Es gibt bereits einen konkreten Fahrplan für die nächsten zwei Jahre. So soll in den nächsten Monaten ein europäisches Klimaschutzgesetz vorgeschlagen werden, mit dem Ziel Klimaneutralität bis 2050 rechtsverbindlich zu verankern. Eine Folgenabschätzung soll noch 2020 darlegen, wie das EU-Ziel für die Reduzierung der Treibhausgasemissionen bis 2030 auf mindestens $50 \%$ und in Richtung 55\% gegenüber 1990 erhöht werden kann. Alle klimabezogenen Politiken sollen bis Mitte 2021 überprüft und gegebenenfalls angepasst werden. Dies betrifft nicht nur das EU-Emissionshandelssystem (EU-EHS), das weite Teile der Energiewirtschaft und der Industrie umfasst, sondern auch die Emissionsminderungsziele der Mitgliedstaaten in den Sektoren außerhalb des Emissionshandelssystems wie Verkehr, Gebäude und Landwirtschaft (Nicht-EHS-Sektoren).

Zurzeit ist das Ziel, die Emissionen im EU-Emissionshandelssystem, das etwas weniger als die Hälfte der Treibhausgasemissionen in der EU abdeckt, bis 2030 um $43 \%$ und in den Sektoren außerhalb des Emissionshandels um insgesamt etwa 30\% gegenüber 2005 zu vermindern. Die Ziele für die Sektoren außerhalb des Emissionshandels sind auf der Ebene der Mitgliedstaaten festgelegt (EU-Lastenteilungsverordnung), für Deutschland sind das $38 \%$. Mit dem Klimaschutzprogramm 2030 möchte die Bundesregierung das deutsche Klimaschutzziel von $55 \%$ weniger Treibhausgasen bis 2030 und die dazugehörigen Sektorziele aus dem Klimaschutzplan erreichen. Gelingen soll dies vor allem mit dem Ausstieg aus der Kohleverstromung bis spätestens 2038 in der Energiewirtschaft und durch die Bepreisung von $\mathrm{CO}_{2}$ in den Sektoren Wärme und Verkehr. Die Planungen in Deutschland scheinen bislang ohne Berücksichtigung des europäischen Green Deals gemacht zu sein. Die Verschärfung des europäischen Klimaziels von $40 \%$ auf $55 \%$ für 2030 wird massive Veränderungen nach sich ziehen müssen - von einem weitgehenden Kohleausstieg in Europa, über eine mehr als Verdoppelung der Erneuerbarenerzeugung, eine überwiegende Elektrifizierung der Wärme, eine fast vollständige Umstellung auf Elektro- und Wasserstofffahrzeuge bis hin zu umfassenden Prozess- und Produktinnovationen in der Industrie. Dabei dürfte die Umsetzung eines gesteigerten EU-Klimaziels nicht nur über eine Verschärfung im EUEmissionshandel erreicht werden, sondern auch durch Anpassungen bei der Lastenteilung und bei zentralen europäischen Maßnahmen etwa den gerade erst beschlossenen europäischen $\mathrm{CO}_{2}$-Standards für Pkw und Lkw oder der EU-Gebäuderichtlinie.

Das langfristige Geschäftsmodell der Kohlekraftwerke war durch die dynamische Entwicklung der erneuerbaren Energien und steigende $\mathrm{CO}_{2}$-Preise im EU-Emissionshandel kaum mehr tragfähig. Viele Kohlekraftwerke sind zudem alt und zunehmend unwirtschaftlich. Fast die Hälfte hätte bis 2030 das Ende ihrer Lebensdauer erreicht, und viele Kraftwerke wären sowieso aus dem Markt ausgeschieden. Der mit den Kraftwerksbetreibern jüngst vereinbarte Ausstiegsfahrplan inklusive hoher Entschädigung für vermeintlich vorzeitiges Abschalten ist vor diesem Hintergrund schwer zu rechtfertigen. Noch schwieriger wird es, wenn es durch den Green Deal zu einer weiteren Verknappung der Emissionszertifikate im

(C) Der/die Autor(en) 2020. Open Access: Dieser Artikel wird unter der Creative Commons Namensnennung 4.0 International Lizenz (https://creativecommons.org/licenses/by/4.0/deed.de) veröffentlicht.

Open Access wird durch die ZBW - Leibniz-Informationszentrum Wirtschaft gefördert.

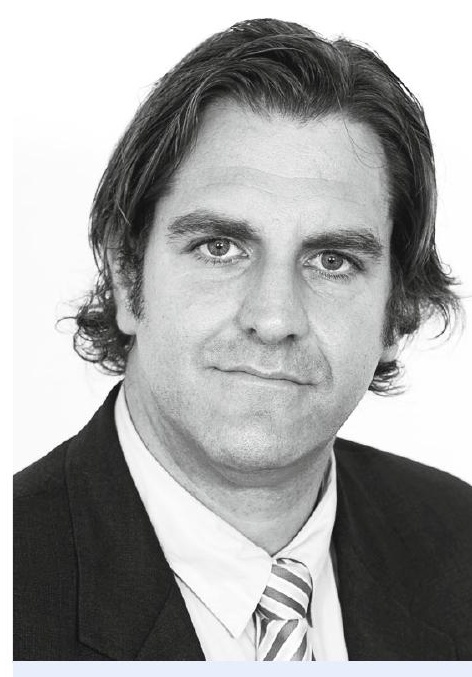

Andreas Löschel ist Professor für Mikroökonomik, insbesondere Energieund Ressourcenökonomik an der Westfälischen Wilhelms-Universität Münster. 
EU-Emissionshandel kommt. Dadurch steigen die $\mathrm{CO}_{2}$-Preise weiter und es kommt zum forcierten marktgetriebenen und kostengünstigen Ausstieg aus der Kohleverstromung - nicht nur in Deutschland. Entschädigungszahlungen für Kraftwerksschließungen, noch dazu weit in der Zukunft, sind nicht nur ökonomisch unnötig, sie könnten sogar schädlich sein, wenn sie die Marktsignale des EU-Emissionshandels abschwächen. Mit einer Verschärfung der Zielsetzungen im europäischen Deal würde es in Deutschland nur noch um mehr Planungssicherheit gehen. Ein (besser) europäischer oder (wenn nötig) nationaler Mindestpreis für $\mathrm{CO}_{2}$ im Emissionshandel stellt den deutschen Kohleausstieg ökonomisch effizient sicher.

Aber auch die Rückkopplung des Green Deals mit den deutschen Anstrengungen im Bereich Wärme und Verkehr ist wichtig. Zur Erreichung der deutschen Minderungsziele soll es eine $\mathrm{CO}_{2}$-Bepreisung in den Sektoren Wärme und Verkehr geben. Dabei ist es gut, einen marktwirtschaftlichen Ansatz zu wählen und den $\mathrm{CO}_{2}$-Preis - sei es nun in Form einer Steuer oder des Emissionshandels - zum Leitinstrument der Klimapolitik zu machen. Er lenkt Investoren und Konsumenten in Richtung klimafreundlicher Entscheidungen bei Wärme und Mobilität. Dazu wird in Deutschland zunächst ein Festpreis für $\mathrm{CO}_{2}$ festgelegt, der von 25 Euro/t CO $\mathrm{CO}_{2}$ (2021) auf 55 Euro/t CO $\mathrm{CO}_{2}$ (2025) steigt. Danach wird die Menge der deutschen Emissionen aus der Verbrennung fossiler Brenn- und Kraftstoffe in einem nationalen Emissionshandelssystem begrenzt, und Emissionszertifikate werden versteigert. Der europäische Grüne Deal dürfte die ökonomische Idee dieses Ansatzes unterlaufen. Statt die unsichtbare Hand des Marktes zu nutzen, um die günstigsten Minderungsoptionen zur Erreichung des deutschen Klimaschutzziels für Wärme und Verkehr zu heben, dürfte es im Rahmen des europäischen Grünen Deals zu umfassenden direkten und teuren Eingriffen kommen, etwa durch die Verschärfung der $\mathrm{CO}_{2}$-Flottengrenzwerte oder der Gebäuderichtlinie.

Daher sollte noch mehr als bisher die Ausweitung des EU-Emissionshandels auf die Bereiche Wärme und Verkehr im Mittelpunkt der deutschen Bestrebungen stehen. Bisher schon gilt, dass sich Vorfestlegungen im Zuge der nationalen Ausgestaltung eines separaten Emissionshandels als problematisch erweisen. Es sollte stattdessen die Ausweitung des EU-Emissionshandelssystems direkt mit den anderen Mitgliedstaaten angegangen werden. Dies sorgt für die dringend notwendige Flexibilisierung. Emissionsreduzierung findet in dem Sektor und dem Mitgliedstaat statt, wo sie am günstigsten zu erreichen ist. Flexibilität bei der Zielerreichung wird auch entscheidend für die Umsetzung des Paris-Abkommens sein. In einer Welt, in der trotzdem weiterhin Unterschiede in den Klimaschutz-Ambitionen bestehen oder sich diese mit dem europäischen Green Deal sogar vergrößern, bedarf es Maßnahmen zum Schutz vor der Verlagerung von $\mathrm{CO}_{2}$-Emissionen. Deshalb ist es richtig, dass im Rahmen des Green Deals auch ein Mechanismus für den Grenzausgleich von $\mathrm{CO}_{2}-$ Preisen für energieintensive Sektoren im internationalen Wettbewerb entwickelt werden soll. Für eine weitgehend klimaneutrale und international wettbewerbsfähige Industrie in Europa wird es aber radikaler Prozess- und Produktinnovationen bedürfen. Noch sind $\mathrm{CO}_{2}-\mathrm{Ab}-$ scheidung und -Speicherung bzw. -Nutzung oder die Erzeugung synthetischer Kraft- und Brennstoffe mit erheblichen Mehrkosten verbunden. Der Aufbau eines Wasserstoffmarkts - der vor allem geeignete Rahmenbedingungen für die Sektorkopplung benötigt - dürfte in der europäischen Industriestrategie eine Schlüsselrolle einnehmen und Teil eines umfassenden Investitionsprogramms sein.

Die Themen für den Green Deal sind gesetzt. Jetzt bietet die deutsche EU-Ratspräsidentschaft im zweiten Halbjahr 2020 die Gelegenheit, den Deal auf europäischer Ebene klug voranzutreiben. So kann die EU das Bekenntnis für ambitioniertere internationale Klimaverhandlungen einlösen und von anderen einfordern. Ende 2020 sollen bei den Klimaverhandlungen in Glasgow erstmals die nationalen Klimaschutzpläne der Staaten unter dem Pariser Klimaschutzabkommen verschärft werden, um das internationale Klimaschutzziel im Auge zu behalten. Schließlich sollte der europäische Grüne Deal ein Anstoß sein, die 